\title{
Improved predictive ability of the Montreal Cognitive Assessment for diagnosing dementia in a community-based study
}

Jung-Lung Hsu ${ }^{1,2,3}$, Yen-Chun Fan ${ }^{4}$, Ya-Li Huang ${ }^{4,5}$, Jui Wang ${ }^{6}$, Wei-Hung Chen ${ }^{7}$, Hou-Chang Chiu ${ }^{7}$ and Chyi-Huey Bai ${ }^{4,5^{*}}$

\begin{abstract}
Introduction: We compared the predictive ability of the Mini-Mental State Examination (MMSE) and the Montreal Cognitive Assessment (MoCA) to diagnose dementia in a community-based study.

Methods: A total of 276 people aged 60 years or older were enrolled. All of the participants were administered face-to-face interview questionnaires and MoCA and MMSE examinations. The receiver operating characteristic curve method and area under curve were performed to assess the predictive ability for diagnosing dementia.

Results: The 276 participants had a mean age of $67.9 \pm 6.1$ years and mean education duration of $11.4 \pm 4.0$ years. In general, the MoCA yielded higher AUCs (0.891) with favorable sensitivity (78 \%) and excellent specificity (94\%) compared with the MMSE in differentiating the participants with and without dementia in either the total sample or all subgroups.
\end{abstract}

Conclusion: Our study determined a higher predictive ability in the MoCA than in the MMSE for diagnosing dementia according to Diagnostic and Statistical Manual of Mental Disorders, Fourth Edition (DSM-IV) criteria in a community-based sample with a broader range of education level.

\section{Introduction}

The population is rapidly aging worldwide $[1,2]$. The prevalence of dementia increases rapidly with age and leads to a burden on public health [3-7]. Detecting moderate cognitive dysfunction early could decrease the risk factors associated with vascular events, resulting in the prevention of dementia [8].

Cognitive function can be measured using several instruments, such as the Clinical Dementia Rating Scale, the Global Deterioration Scale, and the Mini-Mental State Examination (MMSE) [9-12]. The MMSE has been broadly applied to assess cognitive function [13-16], but it is insufficient and highly influenced by education [17]. The Montreal Cognitive Assessment (MoCA) is a new instrument for detecting mild cognitive dysfunction with

\footnotetext{
*Correspondence: baich@tmu.edu.tw

${ }^{4}$ School of Public Health, College of Public Health and Nutrition, Taipei Medical University, 250 Wu-Hsing Street, Taipei City 110, Taiwan

${ }^{5}$ Department of Public Health, College of Medicine, Taipei Medical University, 250 Wu-Hsing Street, Taipei City 110, Taiwan

Full list of author information is available at the end of the article
}

superior sensitivity and can be completed in 10 minutes by clinicians [18].

Numerous studies have validated the cognitive screening ability between the MoCA and MMSE, showing that the MoCA is a more useful screening instrument than the MMSE for detecting dementia [19-24]. Some researchers, however, have indicated that the MoCA was not superior to the MMSE for assessing patients with mild cognitive impairment (MCI) $[25,26]$. In some hospital-based research, the gold standards were the neuropsychological tests [19-23]. Some studies demonstrated their findings in the community, but did not choose a gold standard of the Diagnostic and Statistical Manual of Mental Disorders, Fourth Edition (DSM-IV) [24]. To decide which is best (i.e., diagnosed by physicians according to DSM-IV criteria and community-based samples) is difficult. Thus, the conclusions reported to date remain obscure.

The purpose of this study was to compare the screening ability of the MoCA with that of the MMSE for detecting dementia in a community-based population. In addition, we performed an analysis of subgroups stratified according 
to age, sex, and education level to examine the predictive ability of the MoCA for diagnosing dementia.

\section{Methods}

\section{Study population}

The participants were randomly sampled in 2007-2008 from among all of the residents aged 60 years or older in a community $(n=448)$ that neighbored a teaching hospital. They were invited to participate in a community-based prospective cohort study investigating the cardiovascular and cerebrovascular risk factors in the general population $[27,28]$. The participants were invited again after 3 years to undergo follow-up examinations to measure the neurological assessments from 2011 to 2013. Those who declined to participate in the follow-up study were excluded. A total of 276 remaining individuals were enrolled in this study for the analysis.

\section{Instruments}

The MoCA scale is a cognitive screening test with a high level of interrater reliability and internal consistency for detecting dementia. It can be administered in approximately 10 minutes [18, 20, 22]. Eight subitems were used to measure cognitive function: (1) visuospatial/executive function (an alternation task adapted from the Trail Making Test Part B task, a clock-drawing task, and a three-dimensional cube copy), (2) naming (a three-item confrontation naming task with low-familiarity animals), (3) memory (the short-term memory recall task with two learning trials of five nouns, no points), (4) attention (a sustained attention task with target detection using tapping, a serial subtraction task, and digits forward and backward), (5) language (repetition of two syntactically complex sentences and a phonemic fluency task), (6) abstraction (a twoitem verbal abstraction task), (7) delayed recall (5-minute delayed memory assessment of the short-term memory recall task for five nouns), and (8) orientation (evaluated time and place) [18]. For the participants with less than 12 years of education, the final score was the MoCA plus 1 point. The interrater intraclass correlation coefficient of MoCA in this study was 0.852 , which showed high reliability.

The MMSE test has adequate interrater reliability and internal consistency in predicting dementia. It is used to screen for cognitive impairment and dementia [12].

\section{Clinical evaluations}

The participants were recruited by performing two-stage screening. During the first stage in 2007-2008, a survey with a standardized and structured questionnaire was conducted by well-trained interviewers to obtain demographic information. Age (as a continuous variable and divided into three categories: $60-69,70-79$, and $\geq 80$ years), sex, and duration of education (as continuous variable and divided into three categories: $\leq 6,7-12$, and $>12$ years) were evaluated. An approximately 2-year follow-up survey was conducted to collect the results from a clinical examination performed by neurologists. From 2011 to 2013, all of the participants were administered face-to-face interview questionnaires and the MoCA and MMSE examinations in approximately 30 minutes in clinical practice. The clinical diagnosis of dementia was based on the judgment of a single neurologist. The neurologist made the diagnosis on the basis of interviews of participants and their caregivers (usually the participant's spouse). The participants' previous function was evaluated on the basis of caregivers' and participants' statements. Details were collected regarding cognitive function, such as memory, communication, motor function, recognition or identification of objects, and executive function. Daily activities, including shopping, housekeeping, cocking, doing laundry, and using transportation, were also examined. Next, the office-based neurological examination was performed. If the participant had memory impairment and other domains of cognitive impairment based on the DSM-IV diagnostic criteria for Alzheimer's disease, dementia was diagnosed. The laboratory examination included a blood test, thyroid function examination, and liver and renal function. Brain computed tomography was performed to exclude structural lesions for the possibility of secondary dementia. The neurologist was blinded to participants' cognitive function state.

\section{Statistical analysis}

Statistical analysis was performed using SAS version 9.2 software (SAS Institute, Cary, NC, USA). The descriptive results of continuous variables were expressed as mean \pm standard deviation. Baseline demographic status and MoCA scores were compared by using a $t$ test or oneway analysis of variance for continuous variables and the $\chi^{2}$ test for categorical variables. A $P$ value less than 0.05 was considered statistically significant.

A receiver operating characteristic (ROC) curve was constructed to establish MoCA cutoff points for all of the participants and subgroups. The area under the curve (AUC) was calculated for each of the ROC curves with a $95 \%$ confidence interval. The cutoff scores were derived from the ROC coordinate points, at which both sensitivity and specificity were calculated using Youden's J statistic (Youden's index).

All participants provided written informed consent before study entry. This study was also evaluated and approved by the institutional review board of Taipei Medical University and Shin Kong Wu Ho-Su Memorial Hospital.

\section{Results}

Among the 276 participants, 16 (5.8\%) were diagnosed with dementia and 260 (94.2\%) were diagnosed with normal cognitive function. The demographic variables and MoCA scores of all 276 participants and the subgroups 
were stratified according to sex (Table 1). Compared with the women, the men had a significantly higher duration of education and were older. The MoCA scores for visuospatial function, naming, and abstraction of the men were significantly higher than those of the women. However, no differences in total score by sex were observed.

As shown in Table 2, the MoCA scale scores were stratified according to education level and age. Regarding education level, significant differences were observed between three groups in total scores and the scores for eight subitems.

ROC analysis results for dementia are shown in Table 3 and Fig. 1. For all of the 276 participants, the MoCA yielded greater AUCs than the MMSE to differentiate between participants with and without dementia (0.8913 vs 0.6976). In addition, the MoCA in all subgroups achieved higher AUCs than did the MMSE for dementia. Because no dementia patients were in the high-education group, AUCs could not be calculated.

We determined the cutoff points of the MoCA scale for all participants. The cutoff score on the MoCA was 23.5, demonstrating favorable sensitivity (78 \%) and specificity (94\%).

\section{Discussion}

We show that the MoCA had superior predictive ability for detecting dementia. The sensitivity of 0.78 and specificity of 0.94 of MoCA were higher than the values for the MMSE. For the subgroup analysis, the findings were essentially the same for the women, younger participants (i.e., aged 60-69 years), and those with a low education level. The optimal cutoff score on the MoCA was 23.5, with a high AUC of 0.89 .

In most previous studies [19-24], researchers have also reported that the MoCA appeared to be more useful as a cognitive screening tool than the MMSE. However, the investigators in these studies have not used the same criterion of cognitive dysfunction. The study samples may have been recruited primarily from a clinic and not from the general population, as in our present study [19-23]. In only one study did researchers recruit community-based participants, but the diagnostic criteria were not based on DSM-IV [24]. Other researchers have reported that the MoCA was not more effective than the MMSE in assessing dementia $[25,26]$. The inconsistent findings might be due to the sample location, different participant ages, and varying criteria for diagnosing cognitive function. Distinct sample distributions in different studies make comparing findings difficult.

The cutoff score on the MoCA analyzed for our participants was 23.5, which is consistent with previous studies that indicated a high sensitivity and specificity for identifying Alzheimer's disease and dementia, with optimal cutoff scores ranging from 22 to 26 [19-24]. In some studies, researchers have presented a cutoff point of 23 for the MoCA, similar to our findings, with the average education

Table 1 Comparison of demographic variables and the MoCA scale stratified by gender

\begin{tabular}{|c|c|c|c|c|}
\hline \multirow[b]{3}{*}{ Variables } & \multirow{2}{*}{ Total } & \multicolumn{2}{|c|}{ Gender } & \multirow[b]{3}{*}{$P$ value ${ }^{a}$} \\
\hline & & Male & Female & \\
\hline & $N=276$ & $N=136$ & $N=140$ & \\
\hline Age (years $\pm S D$ ) & $67.93 \pm 6.06$ & $68.93 \pm 6.30$ & $66.95 \pm 5.68$ & $0.0064^{* *}$ \\
\hline Male (\%) & $136(49.28 \%)$ & & & \\
\hline Education (years $\pm S D$ ) & $11.38 \pm 4.01$ & $12.39 \pm 3.98$ & $10.40 \pm 3.82$ & $<0.0001^{* * *}$ \\
\hline Total Score & $25.33 \pm 3.44$ & $25.51 \pm 3.24$ & $25.16 \pm 3.64$ & 0.4088 \\
\hline Visuospatial & $3.97 \pm 1.03$ & $4.15 \pm 0.97$ & $3.81 \pm 1.06$ & $0.0057^{* *}$ \\
\hline Naming & $2.76 \pm 0.57$ & $2.90 \pm 0.35$ & $2.63 \pm 0.69$ & $<0.0001^{* * *}$ \\
\hline Memory1 & $4.03 \pm 1.06$ & $3.98 \pm 1.07$ & $4.08 \pm 1.04$ & 0.4418 \\
\hline Memory2 & $4.61 \pm 0.81$ & $4.55 \pm 0.84$ & $4.67 \pm 0.77$ & 0.3364 \\
\hline Attention1 & $1.85 \pm 0.40$ & $1.85 \pm 0.36$ & $1.84 \pm 0.44$ & 0.8334 \\
\hline Attention2 & $0.95 \pm 0.22$ & $0.94 \pm 0.24$ & $0.96 \pm 0.20$ & 0.5473 \\
\hline Attention3 & $2.85 \pm 0.42$ & $2.85 \pm 0.41$ & $2.84 \pm 0.44$ & 0.8441 \\
\hline Language1 & $1.25 \pm 0.73$ & $1.24 \pm 0.73$ & $1.26 \pm 0.73$ & 0.7415 \\
\hline Language2 & $0.80 \pm 0.40$ & $0.76 \pm 0.43$ & $0.84 \pm 0.37$ & 0.1025 \\
\hline Abstraction & $1.30 \pm 0.76$ & $1.43 \pm 0.75$ & $1.19 \pm 0.76$ & $0.0086^{* *}$ \\
\hline Delayed Recall & $3.04 \pm 1.71$ & $2.98 \pm 1.73$ & $3.11 \pm 1.70$ & 0.5321 \\
\hline Orientation & $5.95 \pm 0.24$ & $5.94 \pm 0.24$ & $5.95 \pm 0.25$ & 0.7632 \\
\hline
\end{tabular}

MoCA Montreal Cognitive Assessment, SD standard deviation

*: $P<0.05 ;{ }^{* *}: P<0.01 ;{ }^{* *}: P<0.0001$

a. Tested by two-sample $t$ test 
Table 2 Comparison of demographic variables and the MoCA scale stratified by education level and age

\begin{tabular}{|c|c|c|c|c|c|c|c|c|c|c|}
\hline \multirow[b]{3}{*}{ Variables } & \multicolumn{5}{|c|}{ Education } & \multicolumn{5}{|c|}{ Age (years) } \\
\hline & Low & Middle & High & & & $60-69$ & $70-79$ & $\geqq 80$ & & \\
\hline & $N=61$ & $N=118$ & $N=97$ & P value ${ }^{a}$ & $P_{\text {trend }}$ & $N=171$ & $N=91$ & $N=14$ & $P$ value $^{a}$ & $P_{\text {trend }}$ \\
\hline Age (years $\pm S D)$ & $67.77 \pm 5.63$ & $68.58 \pm 6.59$ & $67.23 \pm 5.62$ & 0.2572 & 0.4378 & $63.96 \pm 2.91^{\mathrm{a}}$ & $73.27 \pm 2.79^{b}$ & $81.57 \pm 2.44^{c}$ & $<0.0001^{* * *}$ & $<0.0001^{* * *}$ \\
\hline Male (\%) & $20(32.79 \%)$ & 53 (44.92\%) & $63(64.95 \%)$ & $0.0002^{* *}$ & & 77 (45.03\%) & $48(52.75 \%)$ & $11(78.57 \%)$ & $0.0392^{*}$ & \\
\hline Education (years $\pm S D$ ) & $5.66 \pm 1.35^{\mathrm{a}}$ & $10.74 \pm 1.53^{b}$ & $15.76 \pm 1.21^{c}$ & $<0.0001^{* * *}$ & $<0.0001^{* * *}$ & $11.52 \pm 4.10$ & $11.16 \pm 3.96$ & $11.00 \pm 3.33$ & 0.7403 & 0.4450 \\
\hline Total Score & $23.39 \pm 3.87^{a}$ & $25.28 \pm 3.38^{b}$ & $26.62 \pm 2.58^{c}$ & $<0.0001^{* * *}$ & $<0.0001^{* * *}$ & $25.94 \pm 3.09^{\mathrm{a}}$ & $24.62 \pm 3.66^{b}$ & $22.64 \pm 4.09^{b}$ & $0.0001^{* *}$ & $<0.0001^{* * *}$ \\
\hline Visuospatial & $3.38 \pm 1.08^{\mathrm{a}}$ & $3.93 \pm 1.02^{b}$ & $4.40 \pm 0.77^{c}$ & $<0.0001^{* * *}$ & $<0.0001^{* * *}$ & $4.03 \pm 1.00$ & $3.95 \pm 1.02$ & $3.50 \pm 1.34$ & 0.1696 & 0.1116 \\
\hline Naming & $2.59 \pm 0.78^{a}$ & $2.74 \pm 0.55$ & $2.90 \pm 0.37^{b}$ & $0.0032^{* *}$ & $0.0007^{* *}$ & $2.80 \pm 0.52$ & $2.70 \pm 0.62$ & $2.64 \pm 0.74$ & 0.3096 & 0.1281 \\
\hline Memory 1 & $3.62 \pm 1.22^{\mathrm{a}}$ & $4.07 \pm 1.04^{b}$ & $4.25 \pm 0.89^{b}$ & $0.0022^{* *}$ & $0.0008^{* *}$ & $4.15 \pm 1.02$ & $3.86 \pm 1.06$ & $3.50 \pm 1.31$ & $0.0269^{*}$ & $0.0072^{* *}$ \\
\hline Memory2 & $4.41 \pm 0.95^{\mathrm{a}}$ & $4.57 \pm 0.76$ & $4.80 \pm 0.73^{b}$ & $0.0405^{*}$ & $0.0117^{*}$ & $4.64 \pm 0.80^{\mathrm{a}}$ & $4.64 \pm 0.67^{a}$ & $4.10 \pm 1.37^{\mathrm{b}}$ & 0.1198 & 0.1813 \\
\hline Attention 1 & $1.80 \pm 0.44$ & $1.82 \pm 0.45$ & $1.91 \pm 0.29$ & 0.1816 & 0.0855 & $1.88 \pm 0.36^{a}$ & $1.86 \pm 0.38^{\mathrm{a}}$ & $1.43 \pm 0.65^{b}$ & $0.0002^{* *}$ & $0.0050^{*}$ \\
\hline Attention2 & $0.92 \pm 0.28$ & $0.95 \pm 0.22$ & $0.97 \pm 0.17$ & 0.3658 & 0.1602 & $0.96 \pm 0.20^{a}$ & $0.95 \pm 0.23$ & $0.86 \pm 0.36$ & 0.2435 & 0.1686 \\
\hline Attention3 & $2.80 \pm 0.48$ & $2.84 \pm 0.45$ & $2.89 \pm 0.35$ & 0.4668 & 0.2186 & $2.87 \pm 0.38$ & $2.79 \pm 0.51$ & $2.93 \pm 0.27$ & 0.2670 & 0.4875 \\
\hline Language1 & $0.92 \pm 0.78^{\mathrm{a}}$ & $1.18 \pm 0.74^{\mathrm{a}}$ & $1.55 \pm 0.56^{b}$ & $<0.0001^{* * *}$ & $<0.0001^{* * *}$ & $1.35 \pm 0.68^{\mathrm{a}}$ & $1.15 \pm 0.80$ & $0.71 \pm 0.47^{\mathrm{b}}$ & $0.0022^{* *}$ & $0.0008^{* *}$ \\
\hline Language2 & $0.84 \pm 0.37$ & $0.75 \pm 0.43$ & $0.85 \pm 0.36$ & 0.1927 & 0.6787 & $0.86 \pm 0.35^{a}$ & $0.75 \pm 0.44$ & $0.50 \pm 0.52^{b}$ & $0.0011^{* *}$ & $0.0004^{* *}$ \\
\hline Abstraction & $0.79 \pm 0.76^{a}$ & $1.26 \pm 0.76^{b}$ & $1.68 \pm 0.55^{c}$ & $<0.0001^{* * *}$ & $<0.0001^{* * *}$ & $1.40 \pm 0.72^{\mathrm{a}}$ & $1.11 \pm 0.82^{b}$ & $1.36 \pm 0.74$ & $0.0115^{*}$ & $0.0297^{*}$ \\
\hline Delayed Recall & $2.49 \pm 1.76^{\mathrm{a}}$ & $2.94 \pm 1.67^{\mathrm{a}}$ & $3.52 \pm 1.63^{b}$ & $0.0008^{* *}$ & $0.0002^{* *}$ & $3.27 \pm 1.63^{\mathrm{a}}$ & $2.73 \pm 1.81^{b}$ & $2.35 \pm 1.74$ & $0.0149^{*}$ & $0.0039^{* *}$ \\
\hline Orientation & $5.89 \pm 0.37$ & $5.96 \pm 0.20$ & $5.97 \pm 0.17$ & 0.0828 & $0.0472^{*}$ & $5.95 \pm 0.21^{a}$ & $5.97 \pm 0.18^{a}$ & $5.71 \pm 0.61^{b}$ & $0.0010^{*}$ & 0.0560 \\
\hline
\end{tabular}

MoCA Montreal Cognitive Assessment, SD standard deviation

*: $P<0.05$; **: $P<0.01$; ***: $P<0.0001$

a. Tested by ANOVA with Scheffe's test and $x^{2}$ test 
Table 3 AUC and cut-off points of the MoCA, MMSE stratified by gender, education level, and age

\begin{tabular}{|c|c|c|c|c|c|c|c|c|c|c|}
\hline & \multicolumn{5}{|c|}{ MoCA } & \multicolumn{5}{|c|}{ MMSE } \\
\hline & Area & $95 \% \mathrm{Cl}$ & Optimal cut-off & Sensitivity & Specificity & Area & $95 \% \mathrm{Cl}$ & Optimal cut-off & Sensitivity & Specificity \\
\hline Total & 0.8913 & $0.83-0.96$ & 23.5 & 0.78 & 0.94 & 0.6976 & $0.56-0.83$ & 28.5 & 0.38 & 0.92 \\
\hline \multicolumn{11}{|l|}{ Gender } \\
\hline Men & 0.8882 & $0.79-0.98$ & 22.5 & 0.84 & 0.88 & 0.6717 & $0.51-0.84$ & 28.5 & 0.35 & 1.00 \\
\hline Women & 0.8984 & $0.81-0.99$ & 23.5 & 0.76 & 1.00 & 0.7317 & $0.50-0.96$ & 25.5 & 0.77 & 0.67 \\
\hline \multicolumn{11}{|l|}{ Age (years) } \\
\hline $60-69$ & 0.9625 & $0.90-1.00$ & 23.5 & 0.84 & 1.00 & 0.8925 & $0.83-0.96$ & 25.5 & 0.85 & 1.00 \\
\hline $70-79$ & 0.8244 & $0.71-0.94$ & 23.5 & 0.67 & 0.88 & 0.6456 & $0.44-0.85$ & 24.5 & 0.87 & 0.38 \\
\hline$\geqq 80$ & 0.8788 & $0.69-1.00$ & 22.0 & 0.73 & 1.00 & 0.5758 & $0.19-0.96$ & 28.5 & 0.27 & 1.00 \\
\hline \multicolumn{11}{|c|}{ Education level } \\
\hline Low & 0.9709 & $0.91-1.00$ & 20.5 & 0.89 & 1.00 & 0.7965 & $0.60-0.99$ & 24.5 & 0.79 & 0.75 \\
\hline Middle & 0.8465 & $0.75-0.94$ & 23.5 & 0.80 & 0.90 & 0.6290 & $0.45-0.81$ & 28.5 & 0.41 & 0.88 \\
\hline High & & & 22.5 & 0.92 & 1.00 & & & 27.5 & 0.63 & 1.00 \\
\hline
\end{tabular}




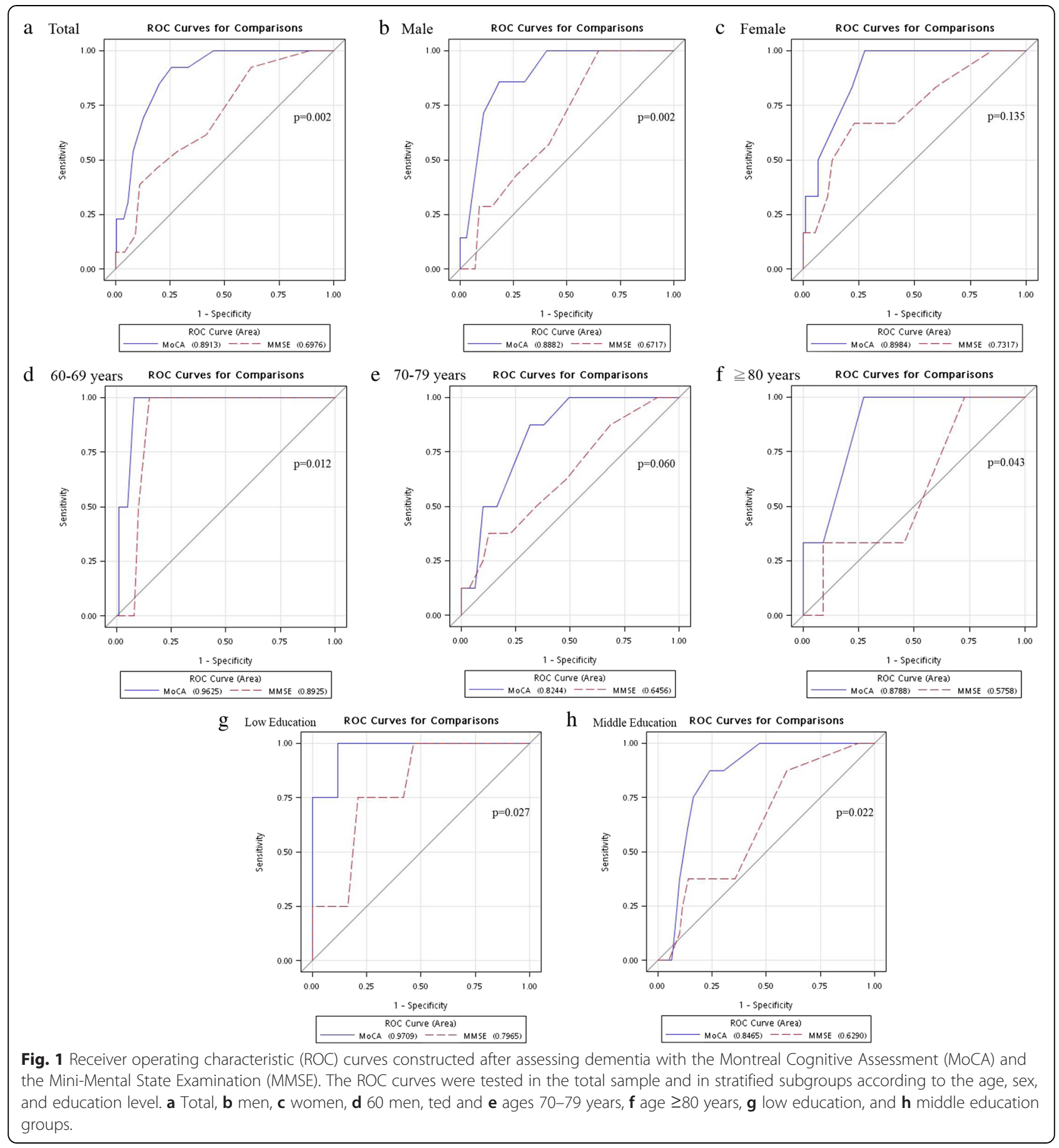

duration ranging from 9.8 to 14.4 years $[19,22,24]$. This means that the MoCA score was not significantly influenced by education level within this range. Furthermore, our results are similar to those reported by Tsai et al., who studied a Taiwanese population [22]. They reported that the optimal cutoff point for dementia was 23 or 24 , with a sensitivity of $92 \%$ and specificity of $78 \%$. These similar results may reflect that both sample populations were close to coherent and the criteria for identifying cognitive impairment were remarkably similar.

In the subgroup analysis, age, sex, and education level were stratified according to the aforementioned definition. The results were largely unchanged. Consistent with previous studies [29, 30], the female participants exhibited slightly higher AUCs. As expected, this suggests that women received shorter education than men did, according 
to their cultural background in China [31]. High MoCA scores were observed for the younger participants and those with a high education level. This result was similar to outcomes in a Chinese-American study [31]. Education and age could influence the MoCA score.

The results for the eight subitems of the MoCA differed in the subgroups of age, sex, and education level. Nonetheless, few studies have investigated the association between subgroups and eight subitems [32, 33]. High scores for visuospatial function, naming, and abstraction among the men were determined in our study. However, this result was not consistent with findings in an Italian study, in which researchers showed that sex influenced the attention and memory domains [32]. The culture difference may explain the disparity in the contrasting results. Our findings regarding the subitem scores of the older participants are comparable to the Italian data, except for the domains of attention and orientation [32]. Another Taiwanese study, published in 2012, showed that the subitem scores on the MoCA were influenced by education level. Participants with 0-6 years of education had the lowest scores for visuospatial and executive function, abstraction, orientation, and naming domains [33].

The strengths of the present study are the use of a community-based population, DSM-IV criteria for diagnosing dementia based on clinical assessments, and analysis of subgroups stratified according to age, sex, and education level. Previous hospital-based sample studies [19-23] led to selection bias, and thus their results cannot be extrapolated to the general population. Dementia was diagnosed by a neurologist according to DSM-IV criteria, and the accuracy was high. Other studies have applied questionnaires to define dementia. Subgroup analysis was also performed in the present study. The results were similar and showed that the MoCA was a stable instrument for assessing dementia. The prevalence of dementia in our study was comparable $(5.8 \%)$ to that in other studies (5.7\% and $5.4 \%)$ done in Taiwan [34, 35]. The representativeness of this communitybased sample is supported.

Several limitations should be carefully addressed. First, the participants were recruited from one district in Taiwan. The representativeness was limited and might not be generalizable to other regions. However, the participants were randomly selected in proportions that were consistent within the population. The discrepancy was then minimized. Second, a substantial proportion of the initially enrolled participants withdrew from the study during the follow-up period. Nevertheless, the difference in demographic distribution between the analyzed sample and those who withdrew was not significant. Third, there were no dementia events in the high education level group, which might have influenced the MoCA cutoff score. Nonetheless, it was similar to that in another Taiwanese study with a MoCA cutoff score of 23/24. Finally, MCI was not assessed in the present study. Diagnoses of MCI are very heterogeneous due to different diagnostic criteria. On the basis of the National Institute on Aging/Alzheimer's Association core clinical criteria for MCI [36], the diagnosis of MCI requires detailed episodic memory measures, such as the Free and Cued Selective Reminding Test or the Logical Memory subtest of the Wechsler Memory Scale. Our study did not include this measurement. A future study will be focused on the relationship between the MoCA score and participants with MCI.

\section{Conclusions}

This study demonstrates that the MoCA had a superior capability to detect dementia compared with the MMSE in a community-based sample with a broad range of education levels. Dementia was diagnosed by a physician according to DSM-IV criteria. Our results not only suggest that the MoCA is a useful screening tool but also explain the different features in the stratified groups. Future studies should be focused on early detection and treatment of cognitive dysfunction in clinical practice.

\section{Abbreviations \\ AUC: area under the curve; DSM-IV: Diagnostic and Statistical Manual of Mental Disorders, Fourth Edition; MCl: mild cognitive impairment; MMSE: Mini-Mental State Examination; MoCA: Montreal Cognitive Assessment; ROC: receiver operating characteristic.}

\section{Competing interests}

The authors declare that they have no competing interests.

\section{Authors' contributions}

JLH made the principal diagnosis of dementia in this study and helped to draft the manuscript. YCF drafted the manuscript and was responsible for the survey work. YLH contributed ideas to the Discussion section and helped to revise the manuscript. JW performed the statistical analysis and was involved in revising the manuscript. WHC and HCC contributed to administrative support for survey work in the hospital, to the interpretation of the results, and to manuscript preparation. CHB was responsible for idea generation, study conception and design, and grant support and helped to draft the manuscript. All authors read and approved the final manuscript.

\begin{abstract}
Acknowledgments
This work was supported by grants from the Taipei Medical University (grant number TMU100-AE1-B08), the National Science Council of Taiwan (grant number NSC-102-2314-B038-034), and the Ministry of Science and Technology of Taiwan (grant number MOST-103-2314-B038-033-MY3).
\end{abstract}

\section{Author details}

${ }^{1}$ Graduate Institute of Humanities in Medicine, Taipei Medical University, Taipei, Taiwan. ${ }^{2}$ Brain and Consciousness Research Center, Taipei Medical University, Shuang Ho Hospital, New Taipei City, Taiwan. ${ }^{3}$ Section of Dementia and Cognitive Impairment, Department of Neurology, Chang Gung Memorial Hospital, Linkou, Taiwan. ${ }^{4}$ School of Public Health, College of Public Health and Nutrition, Taipei Medical University, 250 Wu-Hsing Street, Taipei City 110, Taiwan. ${ }^{5}$ Department of Public Health, College of Medicine, Taipei Medical University, 250 Wu-Hsing Street, Taipei City 110, Taiwan. ${ }^{6}$ Institute of Epidemiology and Preventive Medicine, College of Public Health, National Taiwan University, Taipei, Taiwan. ${ }^{7}$ Department of Neurology, Shin Kong Wu Ho-Su Memorial Hospital, Taipei, Taiwan.

Received: 28 July 2015 Accepted: 13 October 2015

Published online: 09 November 2015 


\section{References}

1. United Nations, Department of Economic and Social Affairs, Population Division. World population prospects: the 2010 revision. Volume I: comprehensive tables. New York: United Nations; 2011. http://www.un.org/ en/development/desa/population/publications/pdf/trends/WPP2010/ WPP2010_Volume-I_Comprehensive-Tables.pdf. Accessed 20 Oct 2015.

2. Hilal S, Ikram MK, Saini M, Tan CS, Catindig JA, Dong YH, et al. Prevalence of cognitive impairment in Chinese: epidemiology of dementia in Singapore study. J Neurol Neurosurg Psychiatry. 2013;84:686-92.

3. Rocca WA, Petersen RC, Knopman DS, Hebert LE, Evans DA, Hall KS, et al. Trends in the incidence and prevalence of Alzheimer's disease, dementia, and cognitive impairment in the United States. Alzheimers Dement. 2011;7:80-93.

4. Prince M, Bryce R, Albanese E, Wimo A, Ribeiro W, Ferri CP. The global prevalence of dementia: a systematic review and metaanalysis. Alzheimers Dement. 2013;9:63-75.e2.

5. Jia J, Wang F, Wei C, Zhou A, Jia X, Li F, et al. The prevalence of dementia in urban and rural areas of China. Alzheimers Dement. 2014;10:1-9.

6. Hurd MD, Martorell P, Delavande A, Mullen KJ, Langa KM. Monetary costs of dementia in the United States. N Engl J Med. 2013;368:1326-34.

7. Zhu CW, Scarmeas N, Ornstein K, Albert M, Brandt J, Blacker D, et al. Health-care use and cost in dementia caregivers: longitudinal results from the Predictors Caregiver Study. Alzheimers Dement. 2015:11:444-54.

8. Kawamura T, Umemura T, Hotta N. Cognitive impairment in diabetic patients: can diabetic control prevent cognitive decline? J Diabetes Investig. 2012;3:413-23.

9. Hughes $C P$, Berg L, Danziger WL, Coben LA, Martin RL. A new clinical scale for the staging of dementia. Br J Psychiatry. 1982;140:566-72.

10. Morris JC. The Clinical Dementia Rating (CDR): current version and scoring rules. Neurology. 1993;43:2412-4.

11. Reisberg B, Ferris SH, de Leon MJ, Crook T. The Global Deterioration Scale for assessment of primary degenerative dementia. Am J Psychiatry. 1982;139:1136-9.

12. Folstein MF, Folstein SE, McHugh PR. "Mini-mental state": a practical method for grading the cognitive state of patients for the clinician. J Psychiatr Res. 1975;12:189-98.

13. Zeki Al Hazzouri A, Vittinghoff E, Byers A, Covinsky K, Blazer D, Diem S, et al. Long-term cumulative depressive symptom burden and risk of cognitive decline and dementia among very old women. J Gerontol A Biol Sci Med Sci. 2014;69:595-601.

14. Matsumoto A, Satoh M, Kikuya M, Ohkubo T, Hirano M, Inoue R, et al. Day-to-day variability in home blood pressure is associated with cognitive decline: the Ohasama study. Hypertension. 2014;63:1333-8.

15. Batty GD, Li Q, Huxley R, Zoungas S, Taylor BA, Neal B, et al. Oral disease in relation to future risk of dementia and cognitive decline: prospective cohort study based on the Action in Diabetes and Vascular Disease: Preterax and Diamicron Modified-Release Controlled Evaluation (ADVANCE) trial. Eur Psychiatry. 2013;28:49-52.

16. Bos D, Vernooij MW, de Bruijn RF, Koudstaal PJ, Hofman A, Franco OH, et al. Atherosclerotic calcification is related to a higher risk of dementia and cognitive decline. Alzheimers Dement. 2015;11:639-47.e1.

17. Kaemmerer T. The influence of age and education on MMSE performance among older adult outpatients with documented memory impairment [abstract A-30]. Arch Clin Neuropsychol. 2014;29:514.

18. Nasreddine ZS, Phillips NA, Bédirian V, Charbonneau S, Whitehead V, Collin I, et al. The Montreal Cognitive Assessment, MoCA: a brief screening tool for mild cognitive impairment. J Am Geriatr Soc. 2005;53:695-9.

19. Gil L, Ruiz de Sánchez C, Gil F, Romero SJ, Pretelt Burgos F. Validation of the Montreal Cognitive Assessment (MoCA) in Spanish as a screening tool for mild cognitive impairment and mild dementia in patients over 65 years old in Bogotá, Colombia. Int J Geriatr Psychiatry. 2015;30:655-62.

20. Yeung PY, Wong LL, Chan CC, Leung JL, Yung CY. A validation study of the Hong Kong version of Montreal Cognitive Assessment (HK-MoCA) in Chinese older adults in Hong Kong. Hong Kong Med J. 2014;20:504-10.

21. Freitas S, Simões MR, Alves L, Santana I. Montreal cognitive assessment: validation study for mild cognitive impairment and Alzheimer disease. Alzheimer Dis Assoc Disord. 2013;27:37-43.

22. Tsai CF, Lee WJ, Wang SJ, Shia BC, Nasreddine Z, Fuh JL. Psychometrics of the Montreal Cognitive Assessment (MoCA) and its subscales: validation of the Taiwanese version of the MoCA and an item response theory analysis. Int Psychogeriatr. 2012;24:651-8.
23. Fujiwara $Y$, Suzuki $H$, Yasunaga $M$, Sugiyama $M$, ljuin $M$, Sakuma $N$, et al. Brief screening tool for mild cognitive impairment in older Japanese: validation of the Japanese version of the Montreal Cognitive Assessment. Geriatr Gerontol Int. 2010;10:225-32.

24. Luis CA, Keegan AP, Mullan M. Cross validation of the Montreal Cognitive Assessment in community dwelling older adults residing in the Southeastern US. Int J Geriatr Psychiatry. 2009;24:197-201.

25. Zhou S, Zhu J, Zhang N, Wang B, Li T, LV X, et al. The influence of education on Chinese version of Montreal cognitive assessment in detecting amnesic mild cognitive impairment among older people in a Beijing rural community. Sci World J. 2014;2014:689456.

26. Kasai M, Meguro K, Nakamura K, Nakatsuka M, Ouchi Y, Tanaka N. Screening for very mild subcortical vascular dementia patients aged 75 and above using the Montreal Cognitive Assessment and mini-mental state examination in a community: the Kurihara project. Dement Geriatr Cogn Dis Extra. 2012;2:503-15.

27. Hsieh $Y C$, Hung $C T$, Lien $L M$, Bai $C H$, Chen $W H$, Yeh $C Y$, et al. A significant decrease in blood pressure through a family-based nutrition health education programme among community residents in Taiwan. Public Health Nutr. 2009;12:570-7.

28. Chou CC, Lien LM, Chen WH, Wu MS, Lin SM, Chiu HC, et al. Adults with late stage 3 chronic kidney disease are at high risk for prevalent silent brain infarction: a population-based study. Stroke. 2011;42:2120-5.

29. Rahman T, El Gaafary MM. Montreal Cognitive Assessment Arabic version: reliability and validity prevalence of mild cognitive impairment among elderly attending geriatric clubs in Cairo. Geriatr Gerontol Int. 2009;9:54-61.

30. Conti S, Bonazzi S, Laiacona M, Masina M, Coralli MV. Montreal Cognitive Assessment (MoCA)-Italian version: regression based norms and equivalent scores. Neurol Sci. 2015;36:209-14.

31. Zheng L, Teng EL, Varma R, Mack WJ, Mungas D, Lu PH, et al. Chineselanguage Montreal Cognitive Assessment for Cantonese or Mandarin speakers: age, education, and gender effects. Int J Alzheimers Dis. 2012;2012:204623.

32. Santangelo G, Siciliano M, Pedone R, Vitale C, Falco F, Bisogno R, et al. Normative data for the Montreal Cognitive Assessment in an Italian population sample. Neurol Sci. 2015;36:585-91.

33. Chang YT, Chang CC, Lin HS, Huang CW, Chang WN, Lui CC, et al. Montreal Cognitive Assessment in assessing clinical severity and white matter hyperintensity in Alzheimer's disease with normal control comparison. Acta Neurol Taiwan. 2012;21:64-73.

34. Wu YT, Lee HY, Norton $\mathrm{S}$, Chen $\mathrm{C}$, Chen $\mathrm{H}, \mathrm{He} \mathrm{C}$, et al. Prevalence studies of dementia in mainland China, Hong Kong and Taiwan: a systematic review and meta-analysis. PLoS One. 2013;8:e66252.

35. Sun Y, Lee HJ, Yang SC, Chen TF, Lin KN, Lin CC, et al. A nationwide survey of mild cognitive impairment and dementia, including very mild dementia, in Taiwan. PLoS One. 2014;9:e100303.

36. Albert MS, DeKosky ST, Dickson D, Dubois B, Feldman HH, Fox NC, et al. The diagnosis of mild cognitive impairment due to Alzheimer's disease: recommendations from the National Institute on Aging-Alzheimer's Association workgroups on diagnostic guidelines for Alzheimer's disease. Alzheimers Dement. 2011;7:270-9.

\section{Submit your next manuscript to BioMed Central and take full advantage of:}

- Convenient online submission

- Thorough peer review

- No space constraints or color figure charges

- Immediate publication on acceptance

- Inclusion in PubMed, CAS, Scopus and Google Scholar

- Research which is freely available for redistribution 